\title{
Blended Chitosan Paste for Infection Prevention: Preliminary and Preclinical Evaluations
}

\author{
Joel M. Berretta MS, Jessica A. Jennings PhD, Harry S. Courtney PhD, \\ Karen E. Beenken PhD, Mark S. Smeltzer PhD, Warren O. Haggard PhD
}

Published online: 17 January 2017

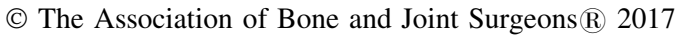

\begin{abstract}
Background Local drug delivery devices offer a promising method for delivering vancomycin and amikacin for musculoskeletal wounds. However, current local delivery devices such as beads and sponges do not necessarily allow for full coverage of a wound surface with eluted antibiotics
\end{abstract}

Research reported in this publication was supported by the National Institute of Arthritis and Musculoskeletal and Skin Diseases of the National Institutes of Health under Award Number R01AR066050 (WOH).

The content is solely the responsibility of the authors and does not necessarily represent the official views of the National Institutes of Health.

All ICMJE Conflict of Interest Forms for authors and Clinical Orthopaedics and Related Research ${ }^{\circledR}$ editors and board members are on file with the publication and can be viewed on request.

Clinical Orthopaedics and Related Research ${ }^{\mathbb{R}}$ neither advocates nor endorses the use of any treatment, drug, or device. Readers are encouraged to always seek additional information, including FDAapproval status, of any drug or device prior to clinical use.

Each author certifies that his or her institution approved the animal protocol for this investigation and that all investigations were conducted in conformity with ethical principles of research. This work was performed at the University of Memphis, Memphis, TN, USA, with the exception of the Zone of Inhibition study, which was performed at the University of Tennessee Health Science Center, Memphis, TN, USA, and the Infection Prevention in a Mouse Pin Model, which was performed at the University of Arkansas for Medical Sciences, Little Rock, AR, USA.

J. M. Berretta ( $\bowtie)$, J. A. Jennings, W. O. Haggard

Department of Biomedical Engineering, The University of

Memphis, 330 Engineering Technology, 3796 Norriswood

Avenue, Memphis, TN 38152, USA

e-mail: jmbrrtta@memphis.edu

H. S. Courtney

Department of Medicine, The University of Tennessee Health

Science Center, Memphis, TN, USA and do not address the need for reducing the antibiotic diffusion distance to help prevent contamination by bacteria or other microorganisms. We blended chitosan/ polyethylene glycol (PEG) pastes/sponges to increase biocompatibility and improve antibiotic coverage within the wound.

Questions/Purposes (1) Are blended chitosan/PEG pastes biodegradable? (2) Are the blended pastes biocompatible? (3) How much force does paste require for placement by injection? (4) Will the pastes elute active antibiotics to inhibit bacteria in vitro? (5) Can the pastes prevent infection in a preclinical model with hardware?

Methods Our blended paste/sponge formulations $(0.5 \%$ acidic, $1 \%$ acidic, and acidic/neutral) along with a control neutral $1 \%$ chitosan sponge were tested in vitro for degradability, cytocompatibility, injectability tested by determining the amount of force needed to inject the pastes, elution of antibiotics, and activity tested using zone of inhibition studies. Along with these studies, in vivo models for biocompatibility and infection prevention were tested using a rodent model and an infected mouse model with hardware, respectively. By evaluating these characteristics, an improved local drug delivery device can be determined.

Results All three of the paste formulations evaluated were almost fully degraded and with 6 days of degradation, the percent remaining being was less than that of the control

K. E. Beenken, M. S. Smeltzer

Department of Microbiology \& Immunology and Department of Orthopaedics, The University of Arkansas for Medical Sciences, Little Rock, AR, USA 
sponge (percent remaining: control $99.251 \% \pm 1.0 \% ; 0.5 \%$ acidic $1.6 \% \pm 2.1 \%, \mathrm{p}=0.002 ; 1 \%$ acidic $1.7 \% \pm 1.6 \%, \mathrm{p}=$ 0.002 ; acidic/neutral $2.3 \% \pm 1.7 \%, \mathrm{p}=0.010)$. There was good biocompatibility because cell viability in vitro was high (control 100.0 $\pm 14.3 ; 0.5 \%$ acidic formulation at $79.4 \pm 12.6$, $\mathrm{p}<0.001 ; 1 \%$ acidic formulation at $98.6 \pm 6.1, \mathrm{p}=0.993$; acidic/neutral formulation at $106.7 \pm 12.8, \mathrm{p}=0.543)$, and in vivo inflammation was moderate (control $2.1 \pm 1.2 ; 0.5 \%$ acidic $3.3 \pm 0.2, \mathrm{p}=0.530 ; 1 \%$ acidic $2.5 \pm 0.9, \mathrm{p}=0.657$; acidic/neutral $2.9 \pm 1.1, \mathrm{p}=0.784$ ). Force required to inject the $0.5 \%$ acidic and $1 \%$ acidic pastes was less than the acidic/ neutral paste used as a control (control $167.7 \pm 85.6 ; 0.5 \%$ acidic $41.3 \pm 10.7, \mathrm{p}=0.070 ; 1 \%$ acidic $28.0 \pm 7.0, \mathrm{p}=0.940)$. At 72 hours, all paste formulations exhibited in vitro activity against Staphylococcus aureus (control $2.6 \pm 0.8 ; 0.5 \%$ acidic $98.1 \pm 33.5, \mathrm{p}=0.002 ; 1 \%$ acidic $87.3 \pm 17.2, \mathrm{p}=0.006$; acidic/neutral $83.5 \pm 14.3, \mathrm{p}=0.010)$ and Pseudomonas aeruginosa (control $163.0 \pm 1.7 ; 0.5 \%$ acidic $85.7 \pm 83.6, \mathrm{p}=$ $0.373 ; 1 \%$ acidic $38.0 \pm 45.1, \mathrm{p}=0.896$; acidic/neutral 129.7 $\pm 78.0, \mathrm{p}=0.896)$. Also, the paste formulations were able to prevent the infection with $100 \%$ clearance on the implanted hardware and surrounding tissue with the control being a $0.5 \%$ acidic paste group without antibiotics (control $4 \times 10^{4} \pm 4.8 \times$ $10^{4} ; 0.5 \%$ acidic $0.0 \pm 0.0, \mathrm{p}$ value: $0.050 ; 1 \%$ acidic $0.0 \pm$ $0.0, \mathrm{p}=0.050$; acidic/neutral $0.0 \pm 0.0, \mathrm{p}=0.050)$.

Conclusions The preliminary studies demonstrated promising results for the blended chitosan/PEG pastes with antibiotics provided degradability, biocompatibility, injectability, and infection prevention for musculoskeletal-type wounds.

Clinical Relevance The preliminary studies with the chitosan paste delivered antibiotics to a contaminated musculoskeletal wound with hardware and prevented infection. More studies in a complex musculoskeletal wound and dosage studies are needed for continued development.

\section{Introduction}

Bacteria such as Staphylococcus aureus and Pseudomonas aeruginosa are common pathogens in musculoskeletal wounds and can form biofilms and gain resistance to certain antibiotic concentrations [2, 6, 7, 10]. To address contaminated injury sites, a local antibiotic delivery system may be utilized to increase the antibiotic levels in the wound to prevent infection without risking patient side effects from high-systemic antibiotic levels [9, 12]. Many types of biomaterials such as bone cement beads and calcium sulfate pellets are being used as local drug delivery devices. These devices can have little or no degradation and need to be removed after use as a local antibiotic delivery device or have rapid degradation that results in sterile wound drainage $[1,9,20]$. Developing a local drug delivery system that could potentially have more compatible degradation byproducts, requires no additional surgeries, and decreases the diffusion distance would be helpful in treating musculoskeletal injuries. Our laboratory has fabricated a chitosan paste blended with polyethylene glycol (PEG) to improve the paste's biocompatibility, biodegradability, antibiotic delivery properties, wound coverage, and diffusion distance (Fig. 1).

Chitosan is a linear aminopolysaccharide that is a product of the process of alkaline deacetylation of chitin from the exoskeletons of crustaceans [1]. Chitosan is biocompatible, biodegradable, and has reactive side groups [1, 3]. Chitosan sponges have been blended with PEG in previous research to aid in degradation and biocompatibility [19]. PEG has known biocompatibility, is used in pharmaceuticals, and helps to increase the biocompatibility and degradability over the initial neutral chitosan sponges [19].

Our goal is to develop a biocompatible local drug delivery device that is able to elute active antibiotics in vivo. In previous studies, chitosan/PEG sponges were developed that were used as an adaptable drug delivery system that is biocompatible, degradable, and can be loaded with antibiotics. However, they were not able to offer complete direct antibiotic exposure to the wound for enhanced infection prevention and treatment [11, 21]. An injectable local delivery system that can fill contours of wounds with deep and/or complex geometry is needed to
Fig. 1 An illustration shows the antibiotic diffusion distances between different biomaterials.
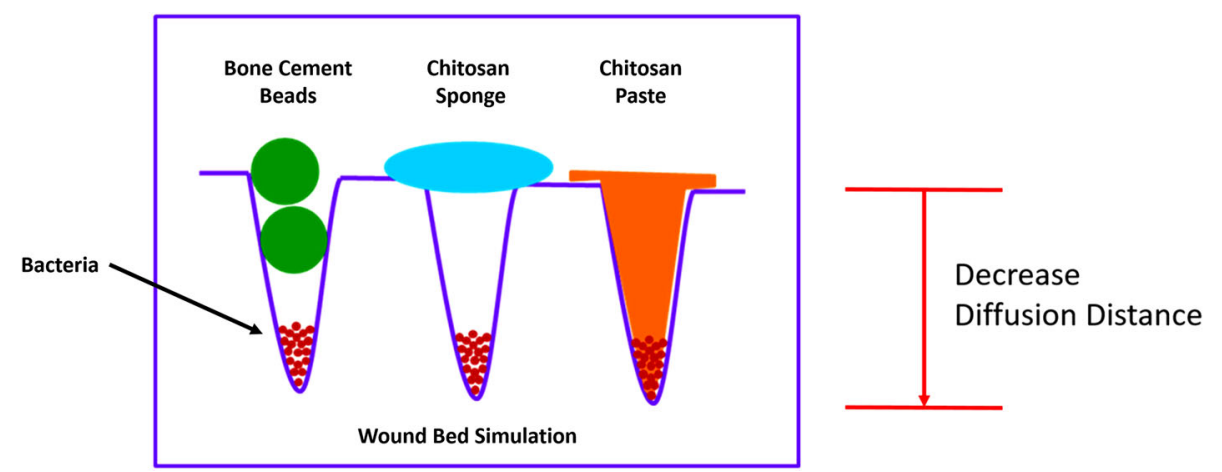
offer enhanced coverage of the wound bed and surface to offer direct exposure to antibiotics.

Therefore, we asked: (1) Are blended chitosan/PEG pastes biodegradable? (2) Are the blended pastes biocompatible? (3) How much force does paste require for placement by manual injection through a modified syringe? (4) Will the pastes elute active antibiotics to inhibit bacteria in vitro? (5) Can the pastes prevent infection in a preclinical model with hardware?

\section{Materials and Methods}

\section{Experimental Overview}

To evaluate these blended chitosan/PEG pastes, multiple in vitro and in vivo studies are needed to determine their effectiveness and clinical utility as local drug delivery systems. Enzymatic degradation, cytocompatibility, paste injectability, elution, and activity studies were assessed through a series of in vitro tests. Finally, two in vivo studies were performed to determine biocompatibility in a

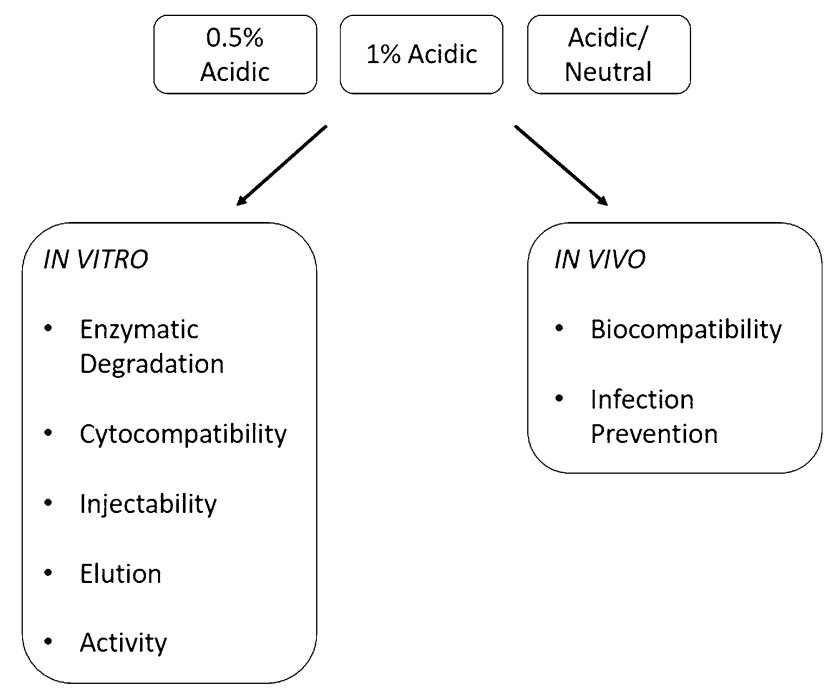

Fig. 2 A flowchart shows the studies performed. rodent model and infection prevention in an infected mouse model (Fig. 2).

\section{Fabrication}

The chitosan/PEG paste was fabricated using Chitopharm ${ }^{\circledR}$ $S$ chitosan powder (Chitinor AS, Troms $\varnothing$, Norway) with an $82^{\circ} \pm 2^{\circ}$ of deacetylation and an average molecular weight of $250 \mathrm{kDA}$. The $1 \%$ acidic paste formulation was made by dissolving $1 \% \mathrm{w} / \mathrm{v}$ of $8000 \mathrm{~g} / \mathrm{mol}$ PEG into a $0.85 \% \mathrm{v} / \mathrm{v}$ of acetic acid in ultrapure water solution and then dissolving $1 \% \mathrm{w} / \mathrm{v}$ of chitosan. The solution is frozen overnight, lyophilized, and then ground up (Table 1 ). The $0.5 \%$ acidic paste is the same with the exception of the amount of PEG being $0.5 \% \mathrm{w} / \mathrm{v}$ of 8000 PEG (Table 1). Finally, the acidic/ neutral paste is composed of equal amounts of an acidic sponge and a neutral sponge. For the neutral portion of the paste, $0.5 \% \mathrm{w} / \mathrm{v}$ of $6000 \mathrm{~g} / \mathrm{mol}$ PEG (Sigma Aldrich, St Louis, MO, USA) was dissolved in a $1 \% \mathrm{v} / \mathrm{v}$ acetic acid in deionized water solution and, after dissolution, $0.5 \% \mathrm{w} / \mathrm{v}$ of chitosan was dissolved in the same solution. The acidic portion of the paste was made in the same manner but by dissolving only $1 \%$ of chitosan alone in the acetic acid solution. The neutral and acidic sponges were cast into $500-\mathrm{mL}$ containers at a volume of approximately $333 \mathrm{~mL}$, frozen overnight at $-20{ }^{\circ} \mathrm{C}$, and lyophilized in a benchtop freeze dryer (LabConco, Kansas City, MO, USA) to create dehydrated sponges. To obtain the neutral sponges, they were immersed in $0.25 \mathrm{M}$ sodium hydroxide and washed with deionized water until a neutral $\mathrm{pH}$ was reached, frozen overnight, and lyophilized again to create the neutral sponges. Both the acidic and neutral sponges were ground separately into small flakes $(\leq 0.3 \mathrm{~cm})$ and then combined in a $1: 1$ weight ratio to be used to make the acidic/neutral paste (Table 1). All samples used for biologic testing were sterilized with ethylene oxide gas $(\mathrm{EtO})$ before hydration and allowed to degas for a minimum of 24 to 48 hours (Fig. 3). To create the paste from the ground up sponge flakes, the samples are hydrated with phosphate-buffered saline (PBS).

Table 1. Compositions of the sponges used to make the acidic/neutral, $0.5 \%$ acidic, and $1 \%$ acidic pastes

\begin{tabular}{|c|c|c|c|c|}
\hline \multicolumn{5}{|c|}{ Types of chitosan sponges used } \\
\hline Type & & Chitosan & PEG & Acetic acid \\
\hline \multirow[t]{2}{*}{ Acidic/neutral } & Combination-acidic chitosan & $1 \%$ & - & $1 \%$ \\
\hline & Combination-neutral chitosan/PEG & $1 \%$ & $0.50 \%$ & $1 \%$ \\
\hline $0.5 \%$ acidic & Acidic chitosan/PEG Type 1 & $1 \%$ & $0.50 \%$ & $0.85 \%$ \\
\hline $1 \%$ acidic & Acidic chitosan/PEG Type 2 & $1 \%$ & $1 \%$ & $0.85 \%$ \\
\hline
\end{tabular}

PEG = polyethylene glycol. 


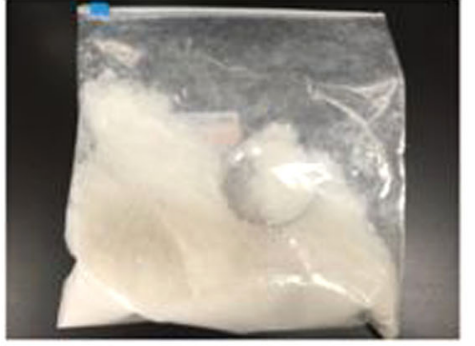

Step 1: Ground Up Chitosan Sponge

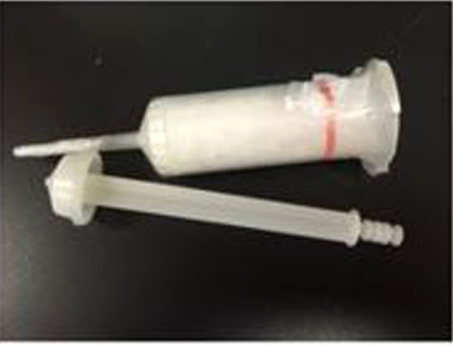

Step 2: Wrap Repeater Pipette Tip in Parafilm, Fill With Amount of Chitosan, and Cover Opening With Parafilm

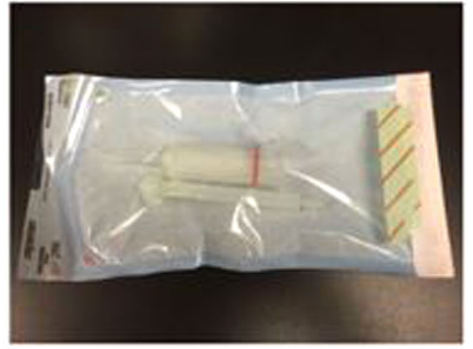

Step 3: Place Repeater

Pipette in Sterilization

Pouch for Eto Sterilization

Fig. 3 Photograph shows the steps for how chitosan paste was prepared for EtO sterilization before being hydrated.

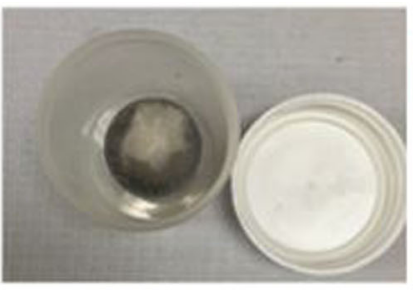

Step 1: Hydrate Paste and Place Inside Metal Hemisphere

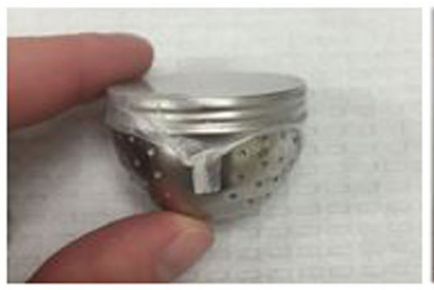

Step 2: Wrap Opening of Metal Hemisphere in Parafilm to Prevent Leakage of Paste/Sponge

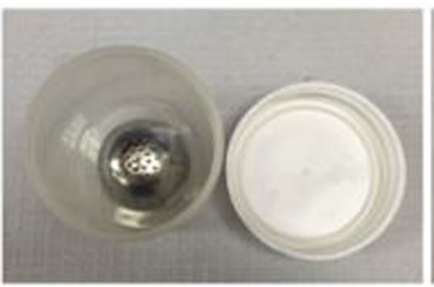

Step 3: Place Wrapped Metal Hemisphere With Paste/Sponge Upside Down Inside Container

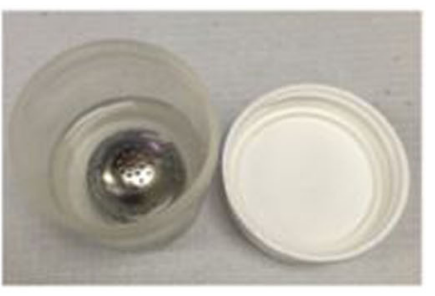

Step 4: Fill Container With 50 $\mathrm{mL}$ of Solution

Fig. 4 Photograph shows the preparation and setup for elution sampling.

\section{Enzymatic Degradation}

To determine biodegradability of the blended chitosan/ PEG pastes, we used an enzymatic degradation experiment to model the process of chitosan degradation inside the body. Lysozyme is the primary enzyme that breaks down chitosan in vivo so for this experiment, we submerged our chitosan pastes in a lysozyme solution to evaluate the degradability of the pastes [19]. The pastes were aliquoted into tubes at approximately $0.67 \mathrm{~g}$ and were then hydrated with $5 \mathrm{~mL}(7.5 \times$ the dehydrated paste weight $)$ of PBS $(\mathrm{n}=$ $6)$. They were then placed into a metal, hemispherical container with numerous holes (approximately $1.5 \mathrm{~mm}$ in diameter) and the opening was wrapped with parafilm while covering none of the holes. The containers were then placed into a 125-mL plastic Nalgene $\mathbb{R}$ ) container (Thermo Fischer Scientific, Waltham, MA, USA), parafilm side down, and filled with $50 \mathrm{~mL}$ of chicken egg white lysozyme solution ( $1 \mathrm{mg} / \mathrm{mL}$ in deionized water) (Fig. 4). The lids were screwed on and the containers were placed inside an incubator at $37{ }^{\circ} \mathrm{C}$ on a shaker (Stovall Belly Dancer Shaker; Cole Palmer, Vernon Hills, IL, USA) at speed 2. Samples were taken at 1-, 2-, 3-, 4-, 5-, and 6-day time points. The lysozyme solution was replaced every 24 hours. The degraded samples were removed from the solution and transferred to an aluminum dish and placed in an oven at $80{ }^{\circ} \mathrm{C}$ for 24 hours to dry. The dried samples were weighed and the percent chitosan/PEG paste remaining was calculated. New samples were used at each time point as a result of the drying process being destructive to the paste.

\section{Cytocompatibility/Biocompatibility}

To evaluate the cytocompatibility and biocompatibility of the chitosan pastes, we used benchtop cell viability experiments to analyze how cultured cells react to these materials and then expanded on this testing using a functional in vivo animal model to assess inflammatory response and tissue reaction. In vitro cytocompatibility was assessed by determining the cell viability of NIH 3T3 cells after being in contact with the pastes and neutral $1 \%$ chitosan sponges (control). The pastes were weighed to be approximately $0.67 \mathrm{~g}$ and were then hydrated with $5 \mathrm{~mL}$ of PBS. Cells were seeded at $1 \times 10^{4}$ cells $/ \mathrm{mL}$ and allowed to proliferate overnight on 24-well transparent tissue culture plates in media under standard cell culture conditions at $37{ }^{\circ} \mathrm{C}$ and $5 \% \mathrm{CO}_{2}$ atmosphere. The media consisted of 0.8 mL High Glucose Dulbecco's Modified Eagle Medium with $10 \%$ fetal bovine serum and $0.1 \mathrm{mg} / \mathrm{mL}$ Normocin 
(InvivoGen, San Diego, CA, USA). Approximately $0.3 \mathrm{~mL}$ of each paste was injected into a cell culture insert $(3.0-\mu \mathrm{m}$ pore size membrane), which were lowered into each well ( $n=5 /$ group). Chitosan sponges were hydrated, placed in inserts, and lowered into the wells. After 24 and 72 hours, the inserts containing the paste were removed and Promega Cell Titer-Glo ${ }^{\circledR}$ Luminescent Cell Viability assay (Promega, Madison, WI, USA) was used to determine cell viability by measuring luminescence at $590 \mathrm{~nm}$ and compared with a standard curve of known quantities of NIH 3T3 fibroblasts.

In vivo compatibility testing was performed for the $0.5 \%$ acidic, $1 \%$ acidic, acidic/neutral, and the neutral $1 \%$ chitosan sponge (control) by quantifying inflammatory responses. The pastes and sponges were weighed to be approximately $0.67 \mathrm{~g}$ and were hydrated with $5 \mathrm{~mL}$ of PBS. The male Wistar rats were anesthetized with isoflurane as anesthesia (UofM-IACUC 0758) [19]. The right hindlimb was shaved and scrubbed with Betadine and isopropanol. An approximate incision of $10 \mathrm{~mm}$ was made with a No. 10 scalpel blade on the mediolateral surface of the leg and the muscle tissue was reflected to expose the proximal tibial surface, which was roughened with a scalpel and the bone site marked for identification. The rats then received implanted paste or sponge ( $n=3 /$ group). Approximately $0.2 \mathrm{~mL}$ of paste was injected into each defect site. Sutures were used to close tissue over the defect followed by a cyanoacrylate application. The rats were monitored until they were awake and active. For 2 days or longer, the rats received an injection of Rimadyl (Zoetis, Parsippany, NJ, USA) for pain management and were checked daily for redness and swelling. After 7 days, the rats were euthanized and the implant region, including the tibia and surrounding tissue, was excised and placed in $10 \%$ neutral formalin before undergoing decalcification and histologic processing with paraffin. Slices were taken perpendicular to the defect.

These slices were then stained with hematoxylin and eosin and the tissue response and amount of remaining paste were determined and quantified by blinded reviewers. The histology was ranked on a scoring system of 0 to $5(0=$ no inflammation, 5 = severe inflammation) [19].

\section{Injectability}

To model clinical application of chitosan paste to a wound site, we performed injection tests through modified syringes to determine the force required for manual injection. Injectability of the pastes was compared by ejecting the paste from a standard $25-\mathrm{mL}$ repeater pipette syringe with a modified 3-mm diameter tip opening with empty syringes used for control $(\mathrm{n}=3)$. The paste was weighed approximately to be $0.8 \mathrm{~g}$ and hydrated in a syringe with 6 $\mathrm{mL}$ of PBS. Each syringe was fixed into an Instron Universal Testing Machine (Instron, Norwood, MA, USA) with a $5-\mathrm{kN}$ load cell automated by Instron's Bluehill ${ }^{\circledR} 2$ (Version 2.13) software, which compressed the syringe plunger at $1 \mathrm{~mm} / \mathrm{sec}$ to fully eject the chitosan paste from the syringe. The maximum ejection force was recorded for comparison. The acidic/neutral paste was used as a control and upper and lower limits were set by personal communication with Wright Medical Technology (Nathan Thomas from Wright Medical Technology, August 2013) and empty syringes (air), respectively.

\section{Elution}

When loading a local drug delivery device with antibiotics, the goal is to release antibiotics at concentrations and locations sufficient to eliminate contaminating bacteria. To evaluate whether the chitosan pastes are releasing antibiotics over a 3-day period, elution studies were conducted with a combination of vancomycin and amikacin antibiotics loaded at concentrations of $10 \mathrm{mg} / \mathrm{mL}$ each. To load the pastes with the antibiotics, the pastes were hydrated with the antibiotic solution. The setup for the pastes and sponges is similar to the enzymatic degradation study except that the pastes and neutral $1 \%$ control sponge were aliquoted into tubes at approximately $0.8 \mathrm{~g}$ and were then hydrated with $6 \mathrm{~mL}(7.5 \times$ the dehydrated paste weight $)$ of the antibiotic solution $(n=3)$ and the containers were filled with $50 \mathrm{~mL}$ of PBS. Samples for antibiotic activity were collected at 1, 3, 6, 12, 24, 48, and 72 hours with the PBS solution replaced at every time point.

Vancomycin concentrations were measured using a Thermo Scientific Dionex UltiMate 3000 Series HPLC and a BDS HYPERSIL reversed-phase C18 column (150 x 4.6 $\mathrm{mm}$ ) (Thermo Fischer Scientific, Waltham, MA, USA). The mobile phase for vancomycin was $30 \%$ acetonitrile and $70 \%$ potassium phosphate buffer. Amikacin concentrations were measured by using an aminoglycoside antibiotics detection method utilizing a reaction solution to read the amount of antibiotics in the samples [13]. All eluate concentrations were normalized to standard curves with known concentrations of the antibiotic solution.

\section{Antimicrobial Activity}

Antimicrobial activity studies followed elution studies to validate concentrations and to ensure that antibiotics are not inactivated by the local delivery system. The activity of the vancomycin and amikacin eluted from the pastes and sponge was determined using zone of inhibition (ZOI) 
assays $(\mathrm{n}=3)$. Blank paper discs, $6 \mathrm{~mm}$ in diameter, were placed on trypticase soy broth (TSB) agar plates containing a lawn of $S$ aureus (ATCC 12598) or $P$ aeruginosa (ATCC 27317) and were hydrated with $20 \mu \mathrm{L}$ of each eluate sample. TSB agar plates were incubated at $37{ }^{\circ} \mathrm{C}$ and removed after 24 hours and photographed. ZOI diameters $(\mathrm{mm})$ were measured.

\section{In Vivo Infection Prevention}

An in vivo implant-associated infection prevention model with orthopaedic hardware was used to determine whether the blended chitosan/PEG pastes loaded with antibiotics are able to prevent an infection in an animal model. We used an infected mouse model to compare the paste formulations and chitosan sponge loaded with antibiotics to a control of the $0.5 \%$ acidic paste loaded with PBS. The paste types evaluated were $1 \%$ acidic, $0.5 \%$ acidic, acidic/ neutral, and the sponge controls (neutral 1\% chitosan sponge), which were all hydrated with an antibiotic solution, a combination of vancomycin and amikacin $(10 \mathrm{mg} /$ $\mathrm{mL}$ each), and then a $0.5 \%$ acidic paste group was hydrated with PBS and used for a control (UAMS-IACUC 3579). The pastes were weighed to be approximately $0.67 \mathrm{~g}$ and both the pastes and the sponge were hydrated with $5 \mathrm{~mL}$ of solution. C57BL/6 mice, 8 to 12 weeks old, were anesthetized with isoflurane and the adequacy of the anesthesia was confirmed by the toe pinch reflex and the reaction to light. The left leg was cleaned with povidine-iodine and rinsed with $70 \%$ ethanol. An incision was made at the knee and a femoral pin (sterile $0.25-\mathrm{mm}$ metal insect pins) was surgically implanted (Fig. 5) [4]. The pin was then inoculated with $2 \mu \mathrm{L}$ of cultures of UAMS- 1 strain of $S$ aureus, a clinical isolate obtained from an osteomyelitis patient ( $S$ aureus [ATCC 49230]). The paste was applied immediately after the implantation of the pin through injection of a volume approximately $0.05 \mathrm{~mL}$ or the neutral sponges were placed adjacent to the pin ( $n=3 /$ group). After suturing, the mice were returned to their cages and monitored daily. Animals were euthanized 7 days after the treatment and the left femur was removed along with the pin, which was then sonicated in PBS. The soft tissue was then dissected from the bone and the femur was cut into small pieces, placed in

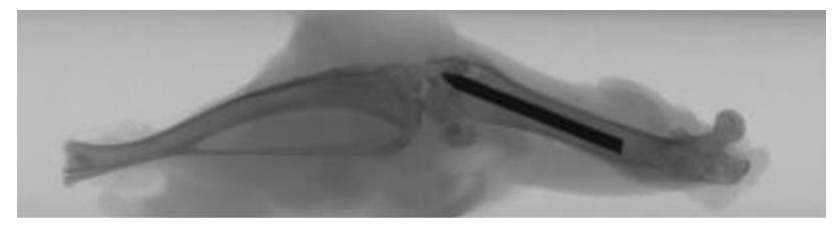

Fig. 5 An image showing the location and placement of the insect pin inside the mouse femur. a sterile saline, and homogenized. The homogenates were diluted, plated on agar plates, and viable microbial colonies were counted along with the colonies remaining on the pin.

\section{Statistical Analysis}

The biodegradability, cytocompatibility, biocompatibility, elution, and activity results were compared using two-way analysis of variance (ANOVA) with the Tukey post hoc test where $\mathrm{p}<0.05$ was considered significant and the mean differences were calculated referenced to the control sponge. When calculating injectability forces, a one-way ANOVA test was used along with the Tukey post hoc test where $\mathrm{p}<0.05$ was considered significant and the mean differences were referenced to the acidic/neutral sponge. For the in vivo infection prevention results, a one-way ANOVA test was used with the Tukey post hoc test where $\mathrm{p}$ $<0.05$ was considered significant and the mean differences were calculated referenced to the $0.5 \%$ acidic paste hydrated with PBS.

\section{Results}

All three of the paste formulations evaluated were almost fully degraded, where by 6 days, the percent remaining was less than that of the control sponge (Table 2; Fig. 6).

The paste formulations show biocompatibility from in vitro and in vivo studies with high cell viability and low inflammatory response (Figs. 7, 8). Cell viability in vitro was high (Table 3; Fig. 7). Also, inflammatory response was comparable in vivo and there was found to be no difference between the control sponge and the paste formulations (Table 4; Figs. 8, 9).

The paste formulations, $1 \%$ acidic and $0.5 \%$ acidic, were found to be injectable with less force when compared with the acidic/neutral paste with upper and lower limits set by a guideline maximum force of $330 \mathrm{~N}$ found from a personal communication from a local medical device company (Nathan Thomas from Wright Medical Technology, August 2013) for similar injectable paste like bone

Table 2. Statistical analysis for enzymatic degradation

\begin{tabular}{llll}
\hline Formulation & $\begin{array}{l}\text { Percent } \\
\text { remaining } \\
(\text { mean } \pm \text { SD) }\end{array}$ & $\begin{array}{l}\text { Mean difference } \\
\text { from control } \\
(95 \% \text { confidence interval) }\end{array}$ & p value \\
\hline Control & $98.9 \pm 1.0$ & & \\
$0.5 \%$ acidic & $1.6 \pm 2.1$ & $97.7(95.2-99.4)$ & 0.002 \\
$1 \%$ acidic & $1.7 \pm 1.6$ & $97.6(95.5-98.9)$ & 0.002 \\
Acidic/neutral & $2.3 \pm 1.7$ & $97.0(94.8-98.4)$ & 0.010 \\
\hline
\end{tabular}




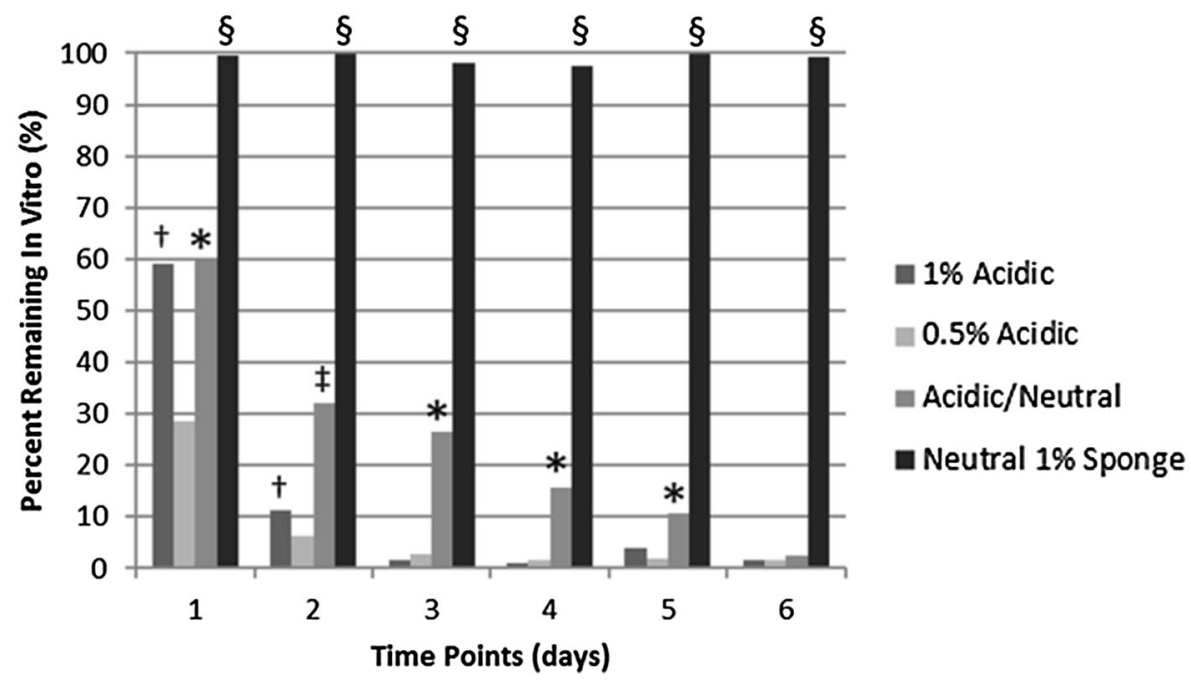

Fig. 6 Enzymatic degradation results are shown for the $1 \%$ acidic, $0.5 \%$ acidic, and acidic/neutral pastes undergoing enzymatic lysozyme degradation $(n=6)$. A significant difference is found whenever $\mathrm{p}<$ 0.05; *significant difference between the acidic/neutral paste and both the $1 \%$ acidic and $0.5 \%$ acidic pastes; ${ }^{\dagger}$ significant difference between the $1 \%$ acidic and $0.5 \%$ acidic pastes; ${ }^{*}$ significant difference between the acidic/neutral paste and the $0.5 \%$ acidic paste; ${ }^{\S}$ historical control from Parker et al. [19]; chitosan/PEG sponges from studies by Parker et al. degraded to between $20 \%$ and $40 \%$ remaining [19].
Fig. 7 In vitro direct contact cytocompatibility, normalized to neutral $1 \%$ chitosan sponge (CS) control, is reported as the average $\pm \mathrm{SD}$ of percent cell viability $(\mathrm{n}=5)$. A significant difference is found whenever $\mathrm{p}$ $<0.05$; *significant difference between the paste and the control sponge; ${ }^{\dagger}$ significant difference between the acidic/ neutral and $0.5 \%$ acidic pastes.

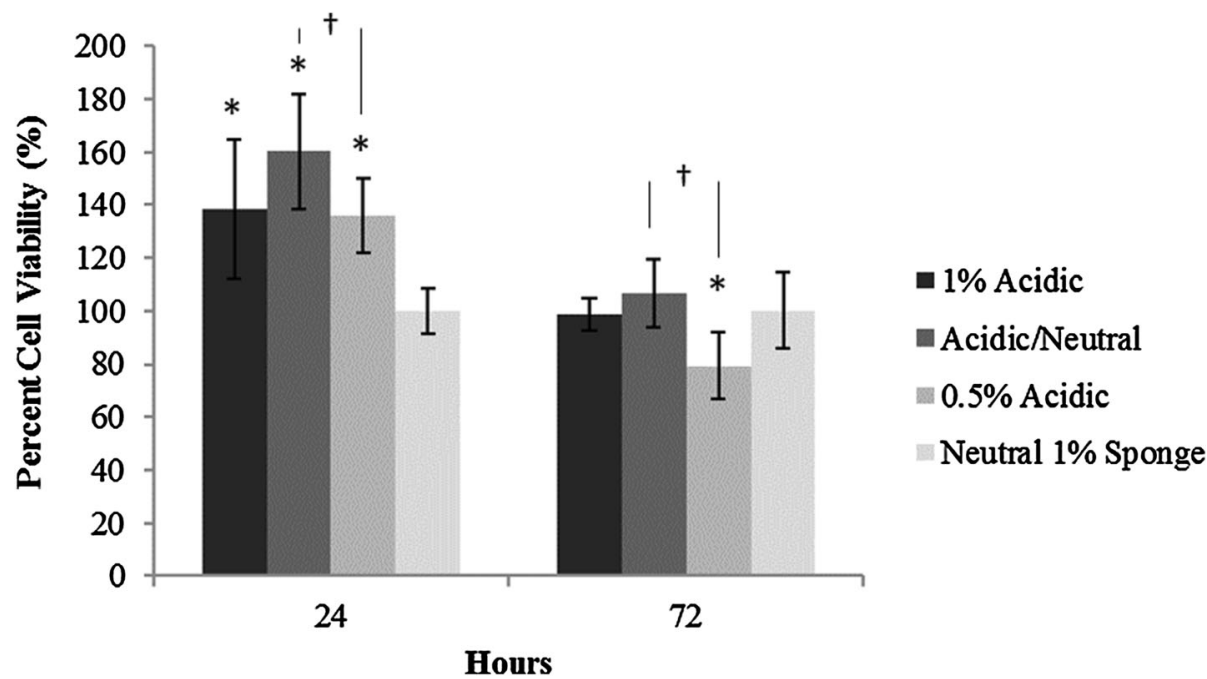

graft products and syringes filled with air (Table 5; Fig. 10).

After 72 hours of the elution study, all samples were shown to be able to elute antibiotics above the minimum inhibitory concentration (MIC) for vancomycin $(2 \mu \mathrm{g} / \mathrm{mL}$ ) and amikacin $(16 \mu \mathrm{g} / \mathrm{mL})$ (Tables 6, 7; Figs. 11, 12).

At 72 hours, all paste formulations exhibited in vitro activity against $S$ aureus (Tables 8A, 9). Similarly, all formulations were effective against $P$ aeruginosa at 72 hours (Tables 8B, 10). However, the control $1 \%$ neutral sponge did not show antibacterial activity against $S$ aureus (Fig. 11) at 72 hours nor against $P$ aeruginosa (Fig. 12) at 24,48 , or 72 hours, although the concentration of eluted antibiotics at these time points was measured to be above the MIC for these bacteria. It is not clear what the cause is of this discrepancy. The assays for measuring the concentrations of antibiotics do not assess functional activity. Thus, some of the released antibiotics in the $1 \%$ neutral sponge may not be functional and this may be more readily detected when the eluted concentrations are lower, after Day 1. It is clear that there was functionally active antibiotics released at the time points with the paste formulations (Table 8).

The antibiotic-loaded $1 \%$ acidic, $0.5 \%$ acidic, and acidic/neutral paste formulations were all able to prevent the infection with $100 \%$ clearance on the implanted 
Fig. 8 Rat tibia model shows results with the neutral $1 \%$ sponge as the control $(\mathrm{n}=3)$. Ranking score: 0 (no inflammation) to 5 (severe inflammation).

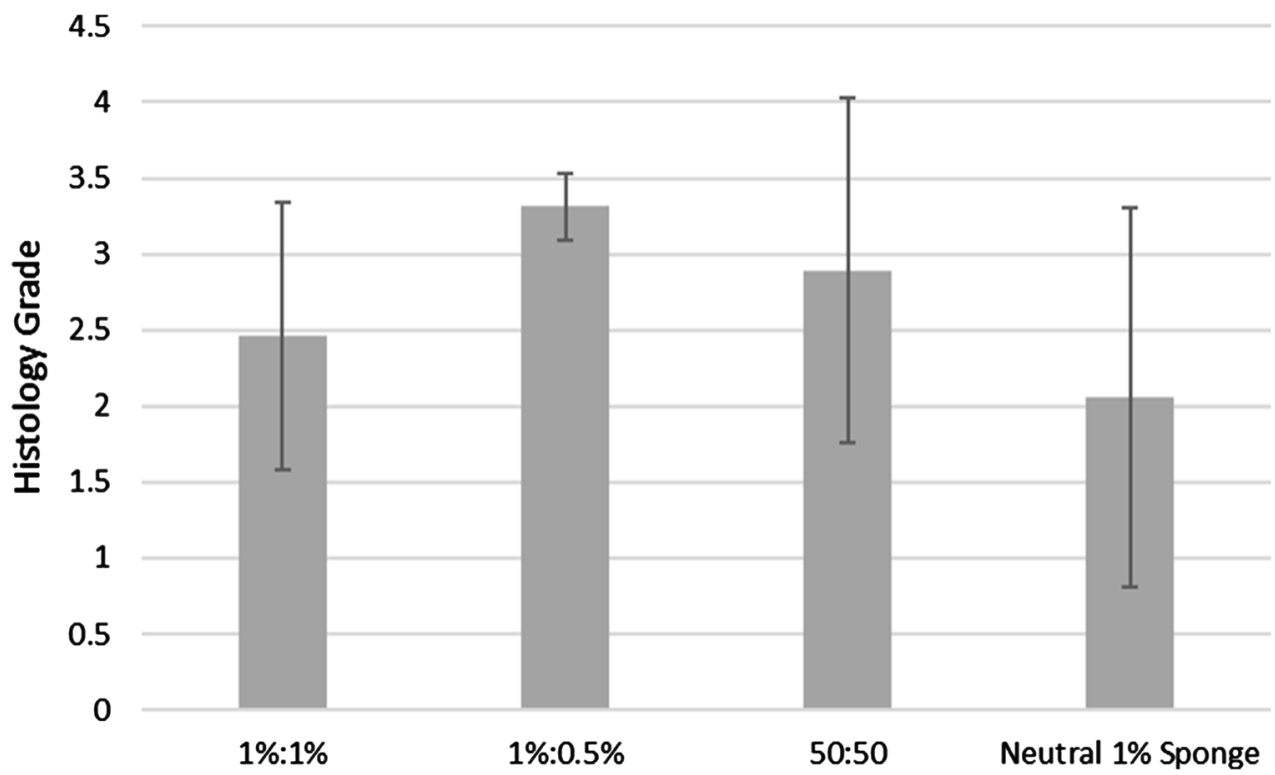

Table 3. Statistical analysis for cytocompatibility

\begin{tabular}{lclr}
\hline Formulation & Cytocompatibility (mean $\pm \mathrm{SD})$ & $\begin{array}{l}\text { Mean difference from control } \\
\text { (95\% confidence interval) }\end{array}$ \\
\hline Control & $100 \pm 14.3$ & & $\mathrm{p}$ value \\
$0.5 \%$ acidic & $79.4 \pm 12.6$ & $20.6(-1.3$ to 42.5$)$ & $<0.001$ \\
$1 \%$ acidic & $98.6 \pm 6.1$ & $1.4(-16.5$ to 19.3$)$ & 0.993 \\
Acidic/neutral & $106.7 \pm 12.8$ & $6.7(-15.4$ to 28.8) & 0.543 \\
\hline
\end{tabular}

Table 4. Statistical analysis for biocompatibility

\begin{tabular}{llll}
\hline Formulation & Biocompatibility $($ mean $\pm \mathrm{SD})$ & Mean difference from control $(95 \%$ confidence interval) & $\mathrm{p}$ value \\
\hline Control & $2.1 \pm 1.2$ & & \\
$0.5 \%$ acidic & $3.3 \pm 0.2$ & $1.2(-1.0$ to 3.4$)$ & 0.530 \\
$1 \%$ acidic & $2.5 \pm 0.9$ & $0.4(-2.4$ to 3.2$)$ & 0.657 \\
Acidic/neutral & $2.9 \pm 1.1$ & $0.8(-2.2$ to 3.8$)$ & 0.784 \\
\hline
\end{tabular}

hardware and surrounding tissue with an antibiotic loading of vancomycin and amikacin solution both at $10 \mathrm{mg} / \mathrm{mL}$ when compared with hydration with PBS (Table 11; Fig. 13).

\section{Discussion}

This preliminary study evaluated a new chitosan-PEG paste local delivery system that would allow for the distribution of eluted antibiotics in a contaminated musculoskeletal wound for infection prevention [16, 18, 22]. Chitosan sponges and chitosan pastes have been investigated for use as local antibiotic delivery systems to address problems of infection and traumatic musculoskeletal injuries [22]. Although local drug delivery devices such as $\mathrm{CaSO}_{4}$ and chitosan sponges offer biocompatibility, degradability, and active antibiotic characteristics, chitosan paste formulations offer an increased distribution surface area for the release of antibiotics allowing for a decreased diffusion distance for the antibiotics to travel before reaching contaminating bacteria in the wound. We asked whether different formulations of chitosan paste would offer increased biocompatibility while maintaining degradability and active antibiotic release over 3 days to develop an improved local drug delivery device to address clinical needs for infection prevention.

Limitations in this preliminary study include minimal proof-of-principle evaluations in both in vivo biocompatibility and functional infection prevention assessment 

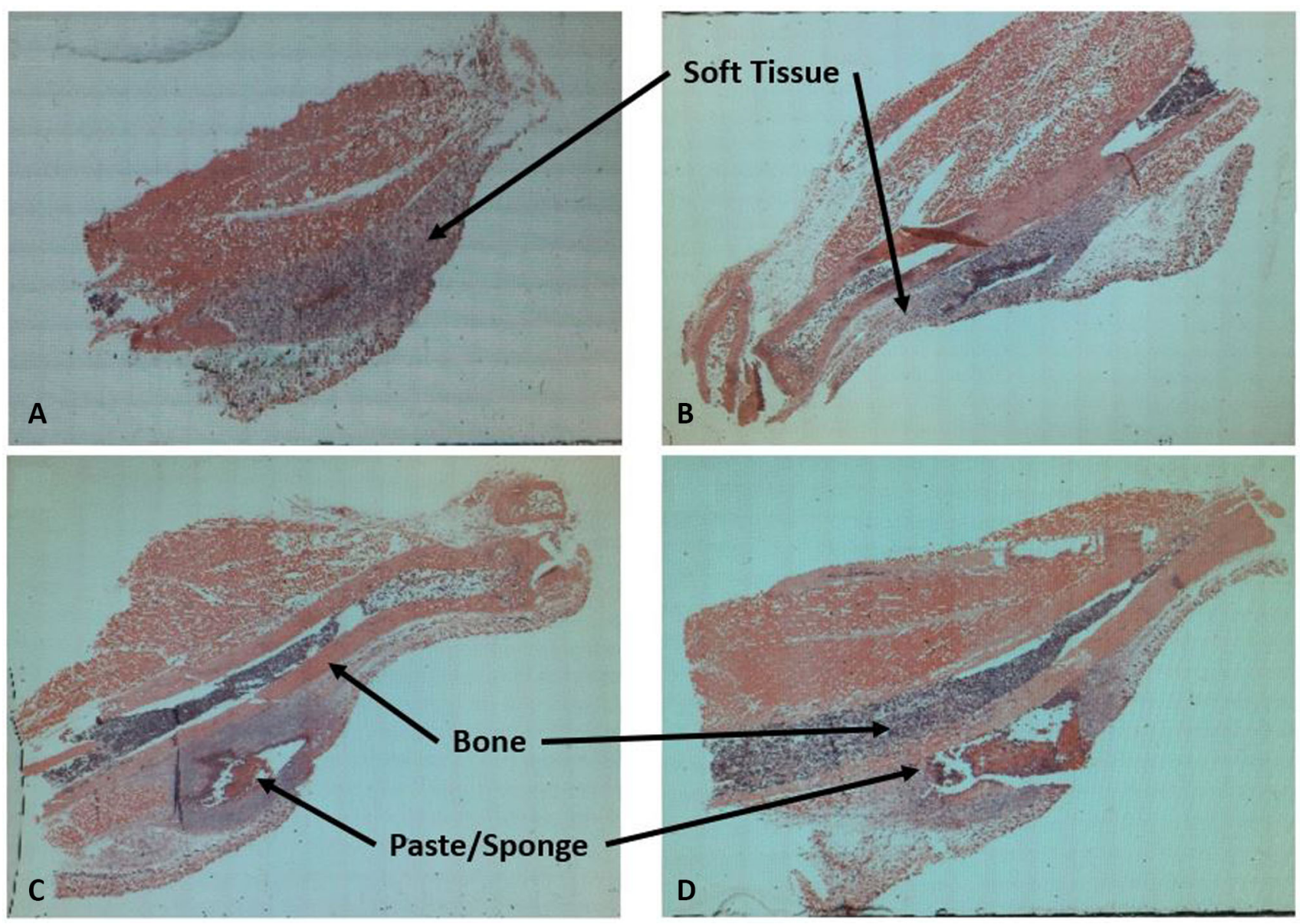

Fig. 9A-D Histology slide examples shown of the rat tibias (Stain, hematoxylin and eosin; original magnification, $\times 160 ;$ A: $1 \%: 1 \%$ paste, B: 1\%:0.5\% paste, C: $50: 50$ paste, D: neutral $1 \%$ sponge).

Table 5. Statistical analysis for injectability

\begin{tabular}{lccc}
\hline Formulation & Force required (mean $\pm \mathrm{SD})$ & $\begin{array}{l}\text { Mean difference from control } \\
\text { (95\% confidence interval) }\end{array}$ \\
\hline Acidic/neutral (control) & $167.7 \pm 85.6$ & & $\mathrm{p}$ value \\
$0.5 \%$ acidic & $41.3 \pm 10.7$ & $126.4(-32.1$ to 284.9) & 0.070 \\
$1 \%$ acidic & $28.0 \pm 7.0$ & $139.7(-18.1$ to 297.5) & 0.940 \\
\hline
\end{tabular}

models. By increasing the in vivo sample size, an enhanced understanding of the biocompatibility and infection prevention qualities could be obtained and these studies are pending or underway. Using a larger animal in a more complex functional infection study would also better represent a bone/soft tissue defect that would be found in clinically infected musculoskeletal wounds. Studies evaluating local antibiotic delivery paste in an established model for infected rabbits is planned. Our main study objective was to improve biocompatibility so the neutral $1 \%$ chitosan sponge was selected for the control based on past biocompatibility results [19]. Expanded control groups using sponges made of the same formulations as the paste would allow for enhanced comparison between sponge versus paste formulations. Despite the discussed limitations, the PEG-blended paste formulations have shown the early potential to be used as local drug delivery devices to treat musculoskeletal wounds and infections. These blended chitosan/PEG pastes have the ability to elute active antibiotics and lessen the antibiotic diffusion distance to contaminating bacteria in complex wounds.

Studies regarding the degradation properties of chitosan/ PEG products and hydrogels involved an injectable, chitosan phospholipid blended with lauric chloride or lauric 
aldehyde for use in localized therapeutic applications. Developed by De Souza et al. [8], the injectable blended hydrogel was tested for degradation in vivo in an intraperitoneally injected mouse model. The results showed that $7.5 \%$ of the injectable hydrogel remained after 4 weeks. Parker et al. [18] used blended chitosan/PEG sponges $(6000 \mathrm{~g} / \mathrm{mol} \mathrm{PEG})$ in a degradation comparison study to a neutral chitosan sponge. After 6 days of lysozyme enzymatic degradation, approximately $60 \%$ of the sponge remained compared with the $0 \%$ to $8 \%$ remaining in the present study. Based on the rapid degradation of our chitosan/PEG paste formulations found in vitro and in vivo, the evaluated pastes in this study have the potential to degrade in vivo over several days to weeks.

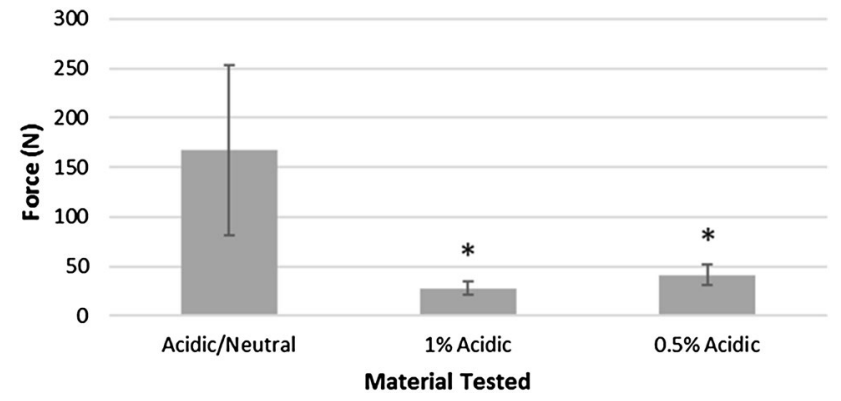

Fig. 10 Injection force results from a modified syringe for the $1 \%$ acidic, $0.5 \%$ acidic, and acidic/neutral paste formulations compared against air $(\mathrm{n}=3)$ are shown. Upper and lower limits $(330 \mathrm{~N}$ and $8 \mathrm{~N}$, respectively) were set by a personal communication with Wright Medical Technology and empty syringes (air). A significant difference is found whenever $\mathrm{p}<0.05$; *significant difference when compared with the acidic/neutral paste.
The chitosan/PEG hydrogel literature has reported acceptable biocompatibility $[14,25,26]$. The results found from this testing performed on chitosan/PEG pastes are comparable to the various reported chitosan hydrogels $[8,17,24]$. Results found from in vivo studies vary from immediate inflammation, tissue encapsulation from an implanted hydrogel, moderate inflammation from chitosan/ PEG devices, to minimal inflammatory response from various hydrogels $[8,14,15,23,26]$. The evaluated blended chitosan/PEG paste formulations in our study experienced similar cellular responses to that of the neutral $1 \%$ chitosan sponge in vitro, which is comparable to the aforementioned studies. From the in vivo biocompatibility study, our pastes elicited a minimal to moderate inflammatory response. These results and past studies suggest that blended chitosan/PEG pastes would elicit a minimal inflammatory response when used clinically in musculoskeletal trauma.

A study has evaluated the injectability of a chitosan/ PEG device. Sprayable thermosensitive chitosan/PEG hydrogels were developed by Wu et al. [26] for nasal drug delivery but they did not explicitly test injectability. The devices in the $\mathrm{Wu}$ et al. [26] study were hydrogels being injected from a needle, whereas our paste was injected from a larger cannula device. This study does not directly reflect our procedures because currently there is not a standardized injectability test. Our results indicate the force required to expel paste from modified syringes would be within the range for manual injection, offering clinical advantages for clinical settings such as the operating room.

Although other chitosan/PEG hydrogels have been shown to release cyclosporine A and bovine serum albumin in a steady release, these hydrogels were not tested for the

Table 6. Statistical analysis for elution of vancomycin at 72 hours

\begin{tabular}{lcll}
\hline Formulation & Activity of vancomycin $($ mean \pm SD) & $\begin{array}{l}\text { Mean difference from control } \\
(95 \% \text { confidence interval) }\end{array}$ \\
\hline Control & $2.6 \pm 0.8$ & & \\
$0.5 \%$ acidic & $98.1 \pm 33.5$ & $95.5(33.9-157.06)$ & 0.002 \\
$1 \%$ acidic & $87.3 \pm 17.2$ & $84.7(53.1-116.3)$ & 0.006 \\
Acidic/neutral & $83.5 \pm 14.3$ & $80.9(54.6-107.2)$ & 0.010 \\
\hline
\end{tabular}

Table 7. Statistical analysis for elution of amikacin at 72 hours

\begin{tabular}{lcll}
\hline Formulation & Activity of amikacin $($ mean $\pm \mathrm{SD})$ & $\begin{array}{l}\text { Mean difference from control } \\
(95 \% \text { confidence interval) }\end{array}$ \\
\hline Control & $163.0 \pm 1.7$ & & $\mathrm{p}$ value \\
$0.5 \%$ acidic & $85.7 \pm 83.6$ & $77.3(-76.3$ to 230.9$)$ & 0.373 \\
$1 \%$ acidic & $38.0 \pm 45.1$ & $125.0(42.1-207.9)$ & 0.053 \\
Acidic/neutral & $129.7 \pm 78.0$ & $33.3(-110.0$ to 176.6$)$ & 0.896 \\
\hline
\end{tabular}




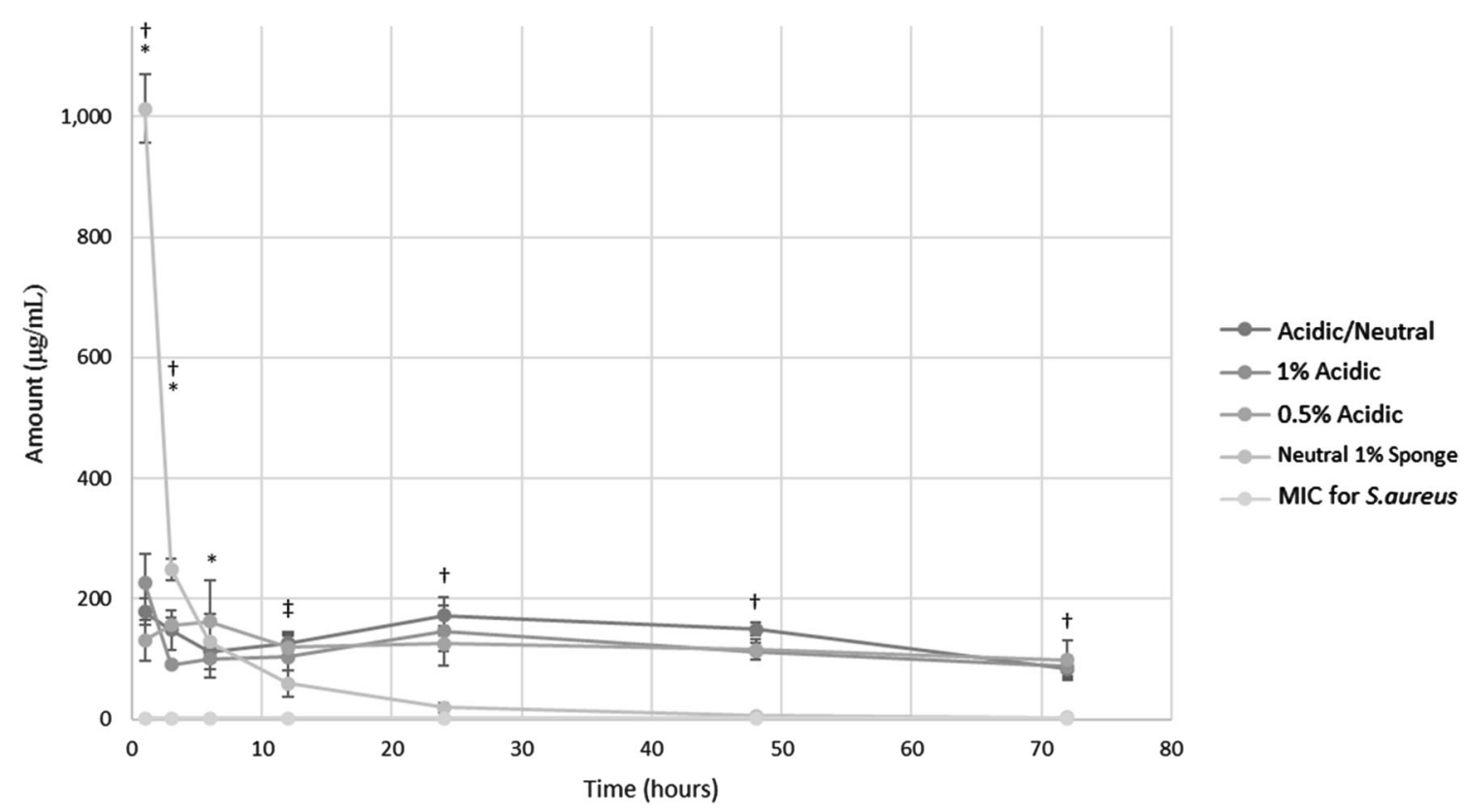

Fig. 11 The elution graph is shown for vancomycin $(n=3)$. A significant difference is found whenever $\mathrm{p}<0.05$; *significant difference between the $1 \%$ acidic and $0.5 \%$ acidic pastes; ${ }^{\dagger}$ significant difference between the neutral $1 \%$ sponge and the $1 \%$ acidic, $0.5 \%$ acidic, and acidic/neutral pastes; ${ }^{*}$ significant difference between the neutral $1 \%$ sponge and the acidic/neutral paste. The MIC for vancomycin is $2 \mu \mathrm{g} / \mathrm{mL}$.

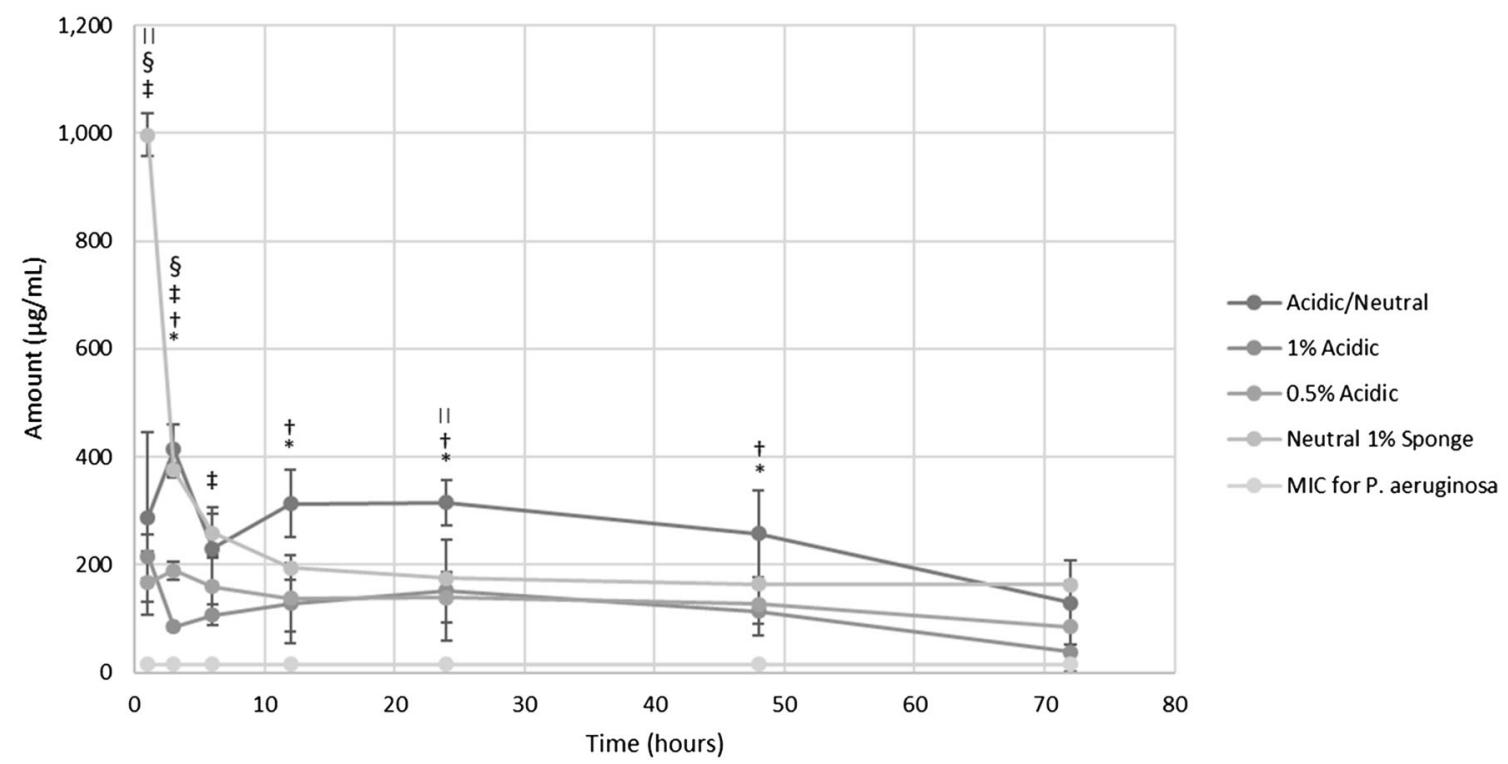

Fig. 12 The elution graph is shown for amikacin $(\mathrm{n}=3)$. A significant difference is found whenever $\mathrm{p}<0.05$; *significant difference between the $1 \%$ acidic and the acidic/neutral pastes; ${ }^{\dagger}$ significant difference between the $0.5 \%$ acidic and acidic/neutral pastes; ${ }^{\star}$ significant difference between the neutral $1 \%$ sponge and the

activity or release of proteins in vitro [5, 14, 25]. Neutral chitosan and chitosan/PEG combination sponges have been shown to release an initial burst release of vancomycin at
$1 \%$ acidic paste; ${ }^{\S}$ significant difference between the neutral $1 \%$ sponge and the $0.5 \%$ acidic paste; "significant difference between the neutral $1 \%$ sponge and the acidic/neutral paste. The MIC for amikacin is $16 \mu \mathrm{g} / \mathrm{mL}$.

$7 \%$ and $16 \%$, respectively, but decreased at longer time points [17]. Chitosan sponges made from acetic and lactic acid loaded with vancomycin reported that after 1 hour, an 
Table 8 Zone of inhibition antibacterial activity results reported as the average $\pm \mathrm{SD}$ of the inhibited growth diameter using a solution combination of vancomycin and amikacin antibiotic eluates against (A) Staphylococcus aureus and (B) amikacin antibiotic eluates

Sample groups Eluate sampling time points (hours) against Pseudomonas aeruginosa from $1 \%$ acidic, $0.5 \%$ acidic, and acidic/neutral chitosan paste formulations after 72 hours of direct contact $(\mathrm{n}=3)$

\begin{tabular}{|c|c|c|c|c|c|c|c|}
\hline & \\
\hline & 1 & 3 & 6 & 12 & 24 & 48 & 72 \\
\hline \multicolumn{8}{|c|}{ A $S$ aureus zone of inhibition diameter $(\mathrm{mm}), \mathrm{n}=3$} \\
\hline $1 \%$ acidic & $16.33 \pm 0.57^{\ddagger}$ & $14.66 \pm 0.57$ & $14.66 \pm 0.57$ & $14.0 \pm 2.0^{*}$ & $15.0 \pm 1.0^{\sharp}$ & $15.0 \pm 0.0^{*}$ & $13.66 \pm 0.57^{*}$ \\
\hline $0.5 \%$ acidic & $15.66 \pm 1.15^{\S}$ & $16.33 \pm 0.57$ & $16.0 \pm 1.73^{*}, \S$ & $14.66 \pm 0.57^{\S}$ & $14.33 \pm 0.57^{\S}$ & $14.0 \pm 1.0^{\S}$ & $12.33 \pm 1.15^{\S}$ \\
\hline Acidic/neutral & $14.33 \pm 0.57^{\prime \prime}$ & $15.33 \pm 0.57$ & $13.33 \pm 0.57^{*}$ & $14.66 \pm 0.57^{\prime \prime}$ & $15.33 \pm 0.57^{\prime \prime}$ & $13.0 \pm 0.0^{\prime \prime}$ & $11.66 \pm 0.57^{\|}$ \\
\hline Neutral $1 \%$ sponge & $19.66 \pm 0.58^{\ddagger, \S, \|}$ & $15.66 \pm 0.58$ & $13.33 \pm 0.58^{\S}$ & $10.66 \pm 1.53^{\ddagger, \S, \|}$ & $8.0 \pm 1.0^{\ddagger, \S, \|}$ & $4.0 \pm 3.46^{\ddagger, \S, \|}$ & $0^{\ddagger}, \S, \|$ \\
\hline \multicolumn{8}{|c|}{ B $P$ aeruginosa zone of inhibition diameter $(\mathrm{mm}), \mathrm{n}=3$} \\
\hline $1 \%$ acidic & $13.33 \pm 0.57^{\ddagger}$ & $10.33 \pm 0.57^{\ddagger}$ & $10.66 \pm 1.52^{\dagger}$ & $10.0 \pm 2.0^{*}$ & $11.66 \pm 1.52^{*}$ & $9.66 \pm 1.52^{*}$ & $7.33 \pm 1.15^{*}$ \\
\hline $0.5 \%$ acidic & $11.66 \pm 1.15^{*, \S}$ & $12.33 \pm 0.57$ & $13.66 \pm 2.08^{\dagger, \S}$ & $11.66 \pm 0.57^{\S}$ & $10.33 \pm 0.57^{\S}$ & $8.66 \pm 1.52^{\S}$ & $6.66 \pm 0.57^{\S}$ \\
\hline Acidic/neutral & $14.66 \pm 0.57^{*, \|}$ & $10.33 \pm 2.08^{\prime}$ & $11.0 \pm 0.0$ & $12.0 \pm 1.0^{\prime \prime}$ & $11.66 \pm 0.57^{\prime \prime}$ & $11.33 \pm 1.15^{\|}$ & $6.33 \pm 0.57^{\prime \prime}$ \\
\hline Neutral $1 \%$ sponge & $18.33 \pm 0.58^{\ddagger, \S, \|}$ & $14.0 \pm 1.0^{\ddagger, \|}$ & $10.0 \pm 1.73^{\S}$ & $4.33 \pm 3.78^{*, \S, \|}$ & $0^{\ddagger, \S, \|}$ & $0^{\ddagger, \S, \|}$ & $0^{\ddagger}, \S$ \\
\hline
\end{tabular}

A significant difference is found whenever $\mathrm{p}<0.05$; * significant difference between the $1 \%$ acidic and acidic/neutral pastes; ${ }^{\dagger}$ significant difference between the $1 \%$ acidic and $0.5 \%$ acidic pastes; ${ }^{*}$ significant difference between the sponge and the $1 \%$ acidic paste; ${ }^{\S}$ significant difference between the sponge and the $0.5 \%$ acidic paste; "significant difference between the sponge and the acidic/neutral paste.

Table 9. Statistical analysis for activity of vancomycin at 72 hours

\begin{tabular}{lcll}
\hline Formulation & Activity of vancomycin $($ mean \pm SD) & $\begin{array}{l}\text { Mean difference from control } \\
(95 \% \text { confidence interval) }\end{array}$ \\
\hline Control & $0.0 \pm 0.0$ & & \\
$0.5 \%$ acidic & $12.3 \pm 1.2$ & $12.3(10.1-14.5)$ & $<0.001$ \\
$1 \%$ acidic & $13.7 \pm 0.6$ & $13.7(12.6-14.8)$ & $<0.001$ \\
Acidic/neutral & $11.7 \pm 0.6$ & $11.7(10.6-12.8)$ & $<0.001$ \\
\hline
\end{tabular}

Table 10. Statistical analysis for activity of amikacin at 72 hours

\begin{tabular}{llll}
\hline Formulation & Activity of amikacin (mean $\pm \mathrm{SD})$ & $\begin{array}{l}\text { Mean difference from control } \\
(95 \% \text { confidence interval) }\end{array}$ \\
\hline Control & $0.0 \pm 0.0$ & & $\mathrm{p}$ value \\
$0.5 \%$ acidic & $6.7 \pm 0.6$ & $6.7(5.6-7.8)$ & $<0.001$ \\
$1 \%$ acidic & $7.3 \pm 1.2$ & $7.3(5.1-9.5)$ & $<0.001$ \\
Acidic/neutral & $6.3 \pm 0.6$ & $6.3(5.2-7.4)$ & $<0.001$ \\
\hline
\end{tabular}

Table 11. Statistical analysis for the infected pin model

\begin{tabular}{lccr}
\hline Formulation & Clearance (mean $\pm \mathrm{SD})$ & $\begin{array}{l}\text { Mean difference from control } \\
\text { (95\% confidence interval) }\end{array}$ \\
\hline Control (PBS) & $39,800 \pm 48,044$ & & $\mathrm{p}$ value \\
$0.5 \%$ acidic & $0.0 \pm 0.0$ & $39,800(-48,463$ to 128,063$)$ & 0.050 \\
$1 \%$ acidic & $0.0 \pm 0.0$ & $39,800(-48,463$ to 128,063$)$ & 0.050 \\
Acidic/neutral & $0.0 \pm 0.0$ & $39,800(-48,463$ to 128,063$)$ & 0.050 \\
Chitosan sponge & $0.0 \pm 0.0$ & $39,800(-48,463$ to 128,063$)$ & 0.050 \\
\hline
\end{tabular}

PBS = phosphate-buffered saline. 
Fig. 13 Remaining microbial colonies from the pin and femur are shown $(\mathrm{n}=3)$. Using ANOVA on ranks and Dunnett post hoc test, a significant difference is found whenever $\mathrm{p}<$ 0.05 ; *significant difference when compared with the pastes and sponges hydrated with the antibiotic solution. CFUs $=$ colony-forming units.

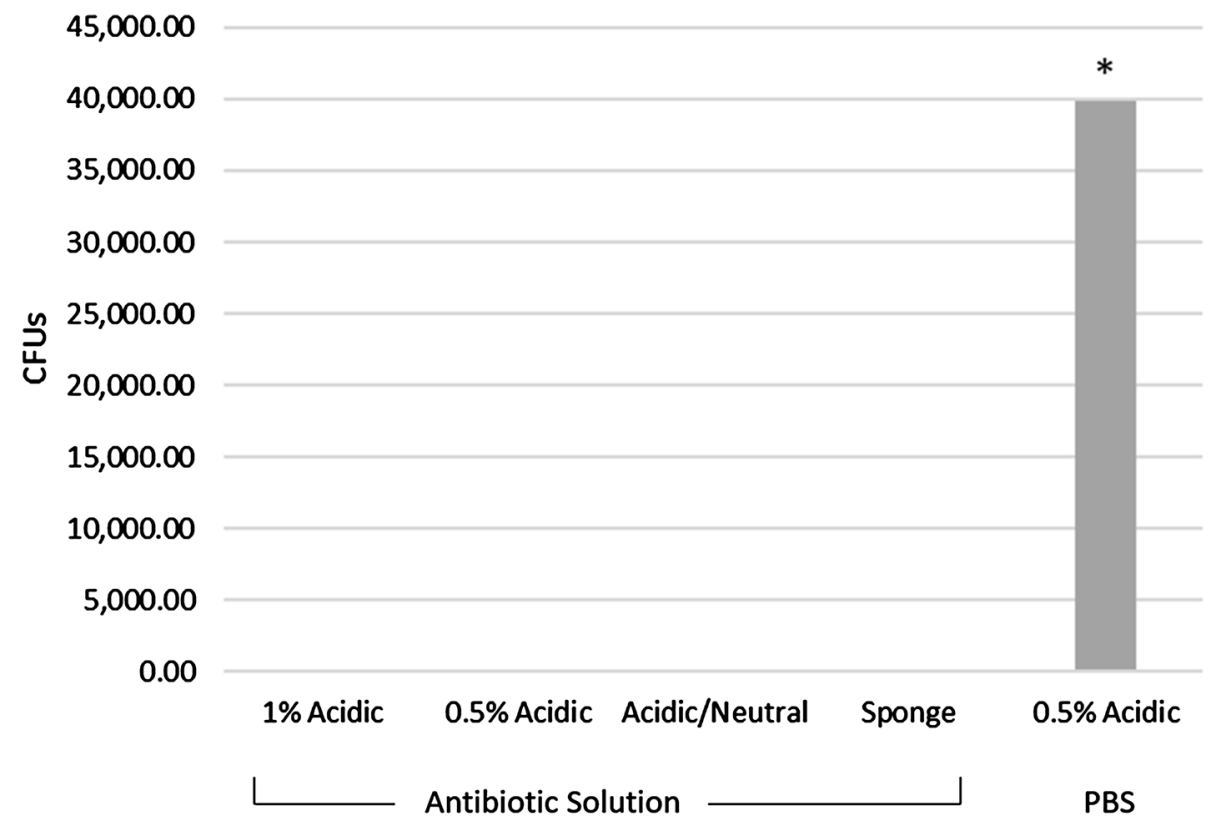

initial burst of antibiotics was recorded with a decrease in antibiotics eluted thereafter [16]. Noel et al. [16] found that the levels of vancomycin and amikacin eluates released from chitosan sponges remained active through hour 72 and hour 48, respectively. The chitosan/PEG pastes that were evaluated in this study showed half the amount of initial antibiotic burst release but did display active antibiotic release through the $72^{\text {nd }}$ hour for both vancomycin and amikacin. Based on the in vivo infected pin model data, this preliminary study illustrates that the pastes can actively release antibiotics and may prevent infection.

The chitosan/PEG pastes being investigated exhibit more rapid degradation and similar biocompatibility to other chitosan/PEG devices as well as showing an extended elution profile of active antibiotics. When tested in vivo in an infected mouse model, the pastes were found to be effective in preventing infections and bacterial growth. Whereas the chitosan sponge may not as effectively match the complex contours of a deep wound, chitosan paste is able to be placed and migrate into the wound bed and release antibiotics closer to the bacteria. In conclusion, the $0.5 \%$ acidic, $1 \%$ acidic, and acidic/neutral blended chitosan/PEG pastes may offer improved characteristics for use as a local drug delivery device to deliver active antibiotics in a wider distribution of eluted antibiotics to prevent or possibly treat musculoskeletal wound and bone infections. Future animal models should be performed to determine the effectiveness of the paste in a more complex musculoskeletal wound along with studies looking at antibiotic loading levels.

Acknowledgments We thank Carlos Wells for his help with HPLC and statistical analysis, Mike Harris and Chris Alexander for help with elution studies, Diego Velasquez Pulgarin for his help with cell culture, and Cheyenne Rhodes and Keaton Smith for their guidance in the laboratory and setting the precedence for this research. This work was conducted in the Biomaterials Applications of Memphis Laboratories at the University of Memphis, Memphis, TN, USA.

\section{References}

1. Agrawal CM. Introduction to Biomaterials: Basic Theory With Engineering Applications. Cambridge Texts in Biomedical Engineering. New York, NY, USA: Cambridge University Press; 2014:xvi, 402.

2. Alanis AJ. Resistance to antibiotics: are we in the post-antibiotic era? Arch Med Res. 2005;36:697-705.

3. Bernstein J, Alonso DR, DiCaprio M, Friedlaender GE, Heckman JD, Ludmerer KM. Curricular reform in musculoskeletal medicine: needs, opportunities, and solutions. Clin Orthop Relat Res. 2003;415:302-308.

4. Bernthal NM, Stavrakis AI, Billi F, Cho JS, Kremen TJ, Simon SI, Cheung AL, Finerman GA, Lieberman JR, Adams JS, Miller LS. A mouse model of post-arthroplasty Staphylococcus aureus joint infection to evaluate in vivo the efficacy of antimicrobial implant coatings. PLOS One. 2010;5:e12580.

5. Bhattarai N, Ramay HR, Gunn J, Matsen FA, Zhang M. PEGgrafted chitosan as an injectable thermosensitive hydrogel for sustained protein release. J Control Release, 2005;103:609-624.

6. Brady RA, Leid JG, Calhoun JH, Costerton JW, Shirtliff ME. Osteomyelitis and the role of biofilms in chronic infection. FEMS Immunol Med Microbiol. 2008;52:13-22.

7. Davis, JS. Management of bone and joint infections due to Staphylococcus aureus. Intern Med J. 2005;35:S79-S96.

8. De Souza R, Zahedi P, Allen CJ, Piquette-Miller M. Biocompatibility of injectable chitosan-phospholipid implant systems. Biomaterials. 2009;30:3818-3824.

9. Diefenbeck M, Muckley T, Hofmann GO. Prophylaxis and treatment of implant-related infections by local application of antibiotics. Injury. 2006;37:S95-S104.

10. Esterhai JL, Gristina AG. Musculoskeletal Infection. Park Ridge, IL, USA: American Academy of Orthopaedic Surgeons; 1992: xvii, 459. 
11. Gitelis S, Brebach GT. The treatment of chronic osteomyelitis with a biodegradable antibiotic-impregnated implant. J Orthop Surg. 2002;10:53-60.

12. Hanssen AD. Local antibiotic delivery vehicles in the treatment of musculoskeletal infection. Clin Orthop Relat Res. 2005;437: 91-96.

13. Jennings JA, Carpenter DP, Troxel KS, Beenken KE, Smeltzer MS, Courtney HS, Haggard WO. Novel antibiotic-loaded pointof-care implant coating inhibits biofilm. Clin Orthop Relat Res. 2015;473:2270-2282.

14. Jiang G, Sun J, Ding F. PEG-g-chitosan thermosensitive hydrogel for implant drug delivery: cytotoxicity, in vivo degradation and drug delivery. J Biomater Sci Polym Ed. 2014;25:241-256.

15. Lih E, Lee JS, Park KM, Park KD. Rapidly curable chitosan/PEG hydrogels as tissue adhesives for hemostasis and wound healing. Acta Biomater. 2012;8:3261-3269.

16. Noel SP, Courtney HS, Bumgardner JD, Haggard WO. Chitosan sponges to locally deliver amikacin and vancomycin: a pilot in vitro evaluation. Clin Orthop Relat Res. 2010;468:2074-2080.

17. Parker AC, Beenken KE, Jennings JA, Hittle L, Shirtliff ME, Bumgardner JD, Smeltzer MS, Haggard WO. Characterization of local delivery with amphotericin B and vancomycin from modified chitosan sponges and functional biofilm prevention evaluation. J Orthop Res. 2014;33:439-447.

18. Parker AC, Jennings JA, Bumgardner JD, Courtney HS, Lindner E, Haggard WO. Preliminary investigation of crosslinked chitosan sponges for tailorable drug delivery and infection control. $J$ Biomed Mater Res B Appl Biomater. 2013;101:110-123.

19. Parker AC, Rhodes C, Jennings JA, Hittle L, Shirtliff, Bumgardner JD, Haggard WO. Preliminary evaluation of local drug delivery of amphotericin B and in vivo degradation of chitosan and polyethylene glycol blended sponges. J Biomed Mater Res B Appl Biomater. 2015;104:78-87.

20. Robinson D, Alk D, Sandbank J, Farber R, Halperin N. Inflammatory reactions associated with a calcium sulfate bone substitute. Ann Transplant. 1999;4:91-97.

21. Smith JK, Bumgardner JD, Courtney HS, Smeltzer MS, Haggard WO. Antibiotic-loaded chitosan film for infection prevention: a preliminary in vitro characterization. J Biomed Mater Res B Appl Biomater. 2010;94:203-211.

22. Stinner DJ, Noel SP, Haggard WO, Watson JT, Wenke JC. Local antibiotic delivery using tailorable chitosan sponges: the future of infection control? J Orthop Trauma. 2010;24:592-597.

23. Sun J, Jiang G, Qiu T, Wang Y, Zhang K, Ding F. Injectable chitosan-based hydrogel for implantable drug delivery: body response and induced variations of structure and composition. J Biomed Mater Res. 2010;95:1019-1027.

24. Tan H, Chu CR, Payne KA, Marra KG. Injectable in situ forming biodegradable chitosan-hyaluronic acid based hydrogels for cartilage tissue engineering. Biomaterials. 2009;30:2499-2506.

25. Tsao CT, Hsiao MH, Zhang MY, Levengood SL, Zhang M. Chitosan-PEG hydrogel with sol-gel transition triggerable by multiple external stimuli. Macromol Rapid Commun. 2015;36: 332-338.

26. Wu J, Wei W, Wang LY, Su ZG, Ma GH. A thermosensitive hydrogel based on quaternized chitosan and poly(ethylene glycol) for nasal drug delivery system. Biomaterials. 2007;28:22202232. 